\title{
OPA1 is Basis for Macrophages, Glucocorticoids Subunits, Neutrophile, RAW264.7 Cells and T-Cells Regulations, where OPA1 Contain COX Sequence Followed by Synthetase, then Synthase, then Phospho- lipase Sequences Respectively
}

\author{
Research Article
}

Volume 1 Issue 2 - 2021

\author{
Author Details \\ Ashraf Marzouk El Tantawi* \\ Biomedical molecular studies, Egypt \\ *Corresponding author \\ Ashraf Marzouk El Tantawi, Biomedical molecular studies, Egypt \\ Article History \\ Received: July 13, 2021 Accepted: July 21, 2021 Published: July 26, 2021
}

\begin{abstract}
OPA1 inner membrane considered as a perfectly regulator tools for glucocorticoid subunits productions (glucocorticoidsgamma $\{\mathrm{GC}-\mathrm{g}\}$ and glucocorticoid-beta $\{\mathrm{GC}-\mathrm{b}\}$ ), RAW264.7 cells, T-cells productions, where OPA1 inner membrane contain COX, then synthetase, followed by synthase, then phospholipase in arranged sequences according to their functions respectively for running and regulating the basis of survival biological pathways in vivo perfectly, where, can be repaired by RORs isoforms productions which originally regulated firstly by ATPase and COX-1 enzymes effect on fatty-nutrients-mTOR molecules for producing long fatty acids chains which will be affected by synthetase, synthase, then by phospholipase for producing the three imp active subunits (gamma subunits, beta-subunits and alpha- subunits respectively).
\end{abstract}

The purpose of this study: Understanding the mitochondrial OPA1 inner membrane function in cellular biological activities for the three RORs isoforms productions, for running biological cycles for producing:

1) Fatty acyl-CoA-synthetase (ROR-gamma),

2) Fatty acyl-CoA synthase (ROR-beta),

3) Fatty acyl-CoA phospholipase (ROR-alpha) synthesis and productions, for phorbol ester which is (fatty- acyl-CoA-synthetase "gamma-subunits") productions, for Toll-like receptor 4 (TLR4) synthesis, for netrin-1 repairs and activities, for LPS-Rs productions, for TLR4 biosynthesis, for reactivating RAW264.7 cells, and for T-cells biosynthesis.

\section{Introduction}

The RORs isoforms and fatty-acyl-CoAs active subunits are comprising the Ser /Thr kinase cascade, where deficiency in Ser amino acids and in pyrimidine synthesis lead to pathogenic in vivo.

fatty-acyl-CoA-synthetase (ROR-gamma) is the basis for RORbeta Subunits productions and for glucocorticoid-gamma and beta synthesis, while Phorbol ester (PMA) can be considered as strong active fatty-acyl-CoA-synthetase biological subunits that depends on the function of synthetase enzyme functions for producing fatty acylCoA-synthetase subunits (gamma- subunits) where both PMA and gamma-subunits are having a necessary roles on carbohydrate and on fat metabolism for producing hydrophobic fatty and amino acids, and both are necessary for acyl-CoA- synthase subunits productions (Beta-subunit) upon synthase effects, then for interleukin beta (ILbeta) productions, for promoting autophagy activities. 
Hypoxia can be defined as a full decreasing in COX or in ATPase enzymes Effects or in gamma oxidation on the absorbed fatty nutrients-mTOR molecules and on inflammations molecules, lead to inhibition in Gamma-subunits synthesis (which is the basis for building proper subunits for carry oxygen and for beta and alpha subunits synthesis) that molecules will not be analyzed properly then will reflect inhibitions in the production of long fatty acids chains that will lead to precipitation in and blockage in capillaries and may can reflect inhibitions in Gamma-oxidations, or in beta-oxidation lead to inhibition in alpha- oxidations.

Fatty-acyl-CoAs Synthetase is the basic effective tool for modulating prostaglandins, for anti- inflammations in availability of proper beta-oxidations, and for improving phospholipid membranes in availabilities of phospholipase functions. Plasmids considered as double stranded subunits gamma-subunits and Beta-subunit that each is running own oxidative processes, where basically plasmids are having the basic origin of the of gamma and beta subunits biosynthesis (fatty-acyl-CoA synthetase and synthase) in vertebrates, that depend on synthetase and synthase enzymes activities in their biosynthesis.

Gamma-oxidations can analyze and inhibit the Generated TLR4 due to over gamma-oxidation process, but beta-oxidations followed by alphaoxidations will activate TLR4 and T-cells functions and activities. (TEAD) transcription factors. has been conserved in proliferating processes such as development, cell growth and proliferation, and it contain a long fatty-acyl-CoA-phospholipase subunits that is necessary for phospholipid synthesis and is fully regulated by both ROR-gamma activity (TAZ subunits) and then by ROR-beta activity (YAP subunits) respectively.

TEAD integrated with and coordinates the Hippo, transforming growth factor beta (TGF- $\beta$ ) started by TGF-gamma which formed by the effects of synthetase then followed by effect of synthase on long fatty acids chains which produced from pro-nutrients-mTOR and from inflammations molecules upon the effects of ATPase and COX enzymes for finally produce TGF-beta subunits that will produce TGF-alpha upon the phospholipase effects.

Hippo pathways is represent the FOX genes effects on same pronutrients-mTOR pathways and can be considered as the main processes for producing the four protein kinases (PSTCK, PSTGk, PSTAk \& PSTT- kinases) which are imp proteins-kinase that produced from the effects of FOX genes and ATPase with mitochondrial OPA1 activities on pro-nutrients-mTOR molecules through secreting mitoenzymes (Cox, Synthetase, synthase, and phospholipase) for acting on lipid, carbohydrate, and proteins, which later will produce three forms of long fatty acyl-CoAs molecules upon the effects of the three mitochondrial enzymes, where those RORs isoforms are the regulator for all biosynthesis pathways and cells proliferations, including beta cells macrophages, and T-cells biosynthesis.

Nuclear hormone receptor retinoic acid receptor-related-orphanreceptor-gamma (ROR- $\gamma$ ) synthesis is depending on synthetase enzymes effects on fatty acids chains and is the basic activator key for transcription factors necessary for re-activating Th17 cell (in availability of synthase functions) and for some of critical cases of IL17 production.

TAZ and YAP signaling processes are the coactivator of TEAD transcription factors, where the core of Hippo pathway and The RORs isoforms biosynthesis are comprising the Ser/Thr kinase cascade. And both of Taz pathway and ROR-gamma (fatty-acyl-CoA synthetase) subunits has the same origin pathways and activities for rebuilding pyrimidine from purines upon their effect on carbohydrate and sugar molecules.

\section{Materials}

- Glucocorticoid subunits (glucocorticoids-gamma $\{\mathrm{GC}-\mathrm{g}\}$ and glucocorticoid-beta $\{\mathrm{GC}-\mathrm{b}\})$,

- RAW264.7 cells,_T-cells

- OPA1 inner membrane

- Retinoic acid receptor-related-orphan-receptor RORs isoforms (ROR- $\gamma+$ ROR-beta +ROR-alpha)

- Fatty-acyl-CoA-synthase (gamma-subunits) +fatty-acyl-CoAsynthase (Beta-subunits) +Fatty-acyl-CoA- phospholipase (alpha subunits)

- $\quad$ T.sub.H17

- Neutrophile

- Three glial mRNAs (glial improved mRNAs): 1_oxidativegamma-RNAs 2_oxidative-beta-RNAs, 3_oxidative-alphaRNAs

- Astrocytes

- Phorbol ester

- $\quad$ PKC membrane

- $\quad$ Toll-like receptor 4 (TLR4),

- Netrin 1

- Bacterial plasmids

- Gamma-subunit and beta-subunits

- $\quad$ LPS-Rs TLR4

\section{Methods and Results}

Transcriptional enhanced associated domain (TEAD) transcription factors play important roles for tissue development, and for cell proliferation. TAZ, is the co-activator of TEAD transcription factors, was expressed under T.sub.H17 cell-inducing conditions and was required for T.sub.H17 differentiation and T.sub.H17 cell-mediated inflammatory diseases [1].

TAZ is critical co-activator of the T.sub.reg cell for activating the pyrimidine resynthesis from purines nucleotides for producing fatty acyl-CoA-synthetase where TAZ has the basic role for digesting carbohydrate, long fatty acids, inflammations, and sugar molecules for adopting purines in tissue (that cooperate with TCA cycles for adopting purines) and in interstitium fluid through producing necessary pyrimidine nucleotides for hydrophobic acids synthesis which is the same pathways for ROR-gamma biosynthesis (Figure 1).

In case of deficiency in necessary hydrophobic acids biosynthesis which carry the critical co-activator role for the T.sub.H17-defining transcription factor will reflect decreasing or inhibition in RORgamma then in ROR-beta subunits productions that will reflect decreasing or inhibition in IL-beta, in TNF-beta and in macrophages Productions, lead to decreasing in the cellular activities and decreasing in T.sub.H17 cell-mediated inflammatory diseases.

In addition, TAZ can prevent or attenuate T.sub.reg cell development till will digest inflammation molecules for ROR-gamma subunits productions upon the effect of synthetase enzymes for promising of converting purine to pyrimidine nucleotides for re-synthesis the necessary hydrophobic acids needed for gamma subunits productions (acyl-CoA synthetase) which will be used for re-produce beta subunits (fatty acyl-CoA synthase) upon synthase effects, where the active gamma-subunits productions which followed by active beta- 
oxidations are necessary for fatty-acyl-CoA-synthase productions for re- activating T.sub.reg cells.

The nuclear hormone receptor retinoic acid receptor-related-orphanreceptor-gamma (ROR- $\gamma$ ) synthesis is depending on synthetase enzymes effects on fatty acids chains, and is the basic activator key for transcription factors necessary for Th17 cell re-activation and for critical cases of the production of IL-17 family of cytokines-beta family which their activities started by the effects of synthase enzyme on the fatty acyl-CoA-gamma subunits for producing fatty acyl-CoAs synthase (or acyl-CoA-beta) subunits for increasing anti-inflammatory effects and immune efficiency that can reflect an adopting in purines and pyrimidines in tissues and in insulin growth (Figure 2).

where, overexpression of ROR-gamma receptors can attenuate or block or reduce the T Helper 17/Interleukin-17 (IL-beta) productions Leading to Attenuated Pro-inflammatory Human Keratinocyte and Skin Responses [2], The relations of the percentage between molecular synthesis of ROR-gamma and ROR-beta have to be in equality and depending in the surrounding cellular environment in vivo.

Where, ROR-gamma can be produced upon synthetase enzyme effects on long fatty acids chains for re- synthesis the hydrophobic acids (whether fatty or amino acids or both) that can lead to attenuating in pro-anti-inflammatory Beta subunits productions which can be associated with the pathogenesis of several inflammatory and autoimmune diseases such as psoriasis, psoriatic arthritis, etc.

RORs isoforms biosynthesis are depending on FOX functions and on mitochondrial OPA1 gene activities, that are comprising the Ser / Thr kinase cascade, While The core Hippo pathway signaling cascade in mammals are comprising serine/threonine kinase cascade too consisting of MST1/2 (homologs of the Drosophila Kinase Hippo), [3] Fatty-acyl-CoA-synthetase (ROR-gamma) is the basis for RORbeta Subunits productions and for glucocorticoid gamma and beta synthesis upon the synthase effects for fatty-acyl-CoA-synthase productions (Figure 3 ).

The TAZ signaling pathway has the same pathway activities as the ROR-gamma (fatty-acyl-CoA synthetase) subunits biosynthesis pathway, that the increasing in TAZ signaling processes will raise up levels of carbohydrate and fatty acids metabolism and adopt (or decrease) purines in blood levels, while will raise the pyrimidine synthesis and maintain the proper balance of purines and pyrimidines for hydrophobic acids synthesis for active gamma subunits synthesis, (fatty acyl-CoA synthetase) that can be used for re-activating glucocorticoid-gamma productions, while the effects of synthase on acyl-CoA- synthetase (gamma subunits) will produce glucocorticoidbeta which can produce IL-beta that can reactivate astrocyte, netrin-1, and neutrophiles functions.

The glucocorticoid Receptor (GRs) has roles of function activating steroid hormone, and mitochondrial matrix repair and activations, where glucocorticoid (GC) contains the estrogen receptor that GR has strong roles for estrogen biosynthesis and strong roles in antiinflammatory pathways.

The glucocorticoids (GCs) Biosynthesis pathways is linked and regulated by synthetase effects on phosphorylated long fatty acids chains for glucocorticoid-gamma (gamma subunits) productions followed by synthase enzyme effects for producing glucocorticoidbeta subunits productions (beta subunits) which is basic units for netrin-1 synthesis which are the basic for the neutrophile repairs and reactivations.

Glucocorticoids biological molecules are carrying two imp roles of 1st, pro-inflammatory subunits productions, which results from the effects of ROR-gamma (effect of fatty-acyl-CoA-synthetase enzymes) on inflammatory molecules, 2nd, anti-inflammatory activities due to production of IL-beta and beta subunits for acting on inflammation molecules.

Glucocorticoid-gamma has anti-inflammatory activities on inflammations for producing pro- inflammatory subunits due to the effects of acyl-CoA synthetase (gamma subunits) on inflammations for analysing their contents for starting anti-inflammatory pathways activity.

While glucocorticoid-beta is attributed to the repression of proinflammatory subunits through signal transduction by their steroid beta subunits (beta oxidations). That glucocorticoid can have two basics subunits:

\section{Glucocorticoid gamma,}

\section{Glucocorticoids-beta.}

Both Glucocorticoids (GC) gamma and beta subunits are essential for regulating T-cell development but in availability of phospholipase activities [4]. glucocorticoids have been described to promote Th2 cell predominance [5]

Glucocorticoids can be found in nature as remnants of vertebrates and dead animals, that appear in nature as microorganisms consisting of two subunits as bacterial plasmids or as viral one beta subunits strand self-replicated, that when infected humans firstly will cause pro-inflammation molecules or subunits upon the effects of gamma subunits followed by beta oxidations process upon the effects of bacterial beta subunits that can replicated to form protein as antigens surrounding host cells then in pathogenesis can cause invasion, which can involve enzymes and toxins during bacterial self-replication.

As Glucocorticoids (GCs) can also affect antigen presentation [6]. As bacteria and microorganisms are having the GC origin as will effect on antigen presentation too. In addition, TAZ signaling pathway coordinate hepatic glucose, where TAZ coordinates hepatic glucose homeostasis in normal physiological states [7], that TAZ signaling process has the role of functions of adopting purines for pyrimidine synthesis in vivo. In addition, TAZ/YAP signaling pathway is inactivated under the low glucose condition or by the inhibition of glycolysis while it is activated in response to high levels of glucose metabolism $[8,9]$.

The TAZ signaling process is depending on synthetase enzyme activities (which has the imp roles in carbohydrate metabolism) for producing fatty-acyl-CoA-synthetase (ROR-gamma) isoforms synthesis, while YAP signaling process is the effects of synthase enzyme on ROR-gamma subunits (on fatty acyl- CoAs synthetase) for produce acyl-CoA synthase (ROR-beta) subunits or fatty-acyl-CoAsynthase, which regulate netrin-1 productions which has the basis of neutrophile synthesis and repairs which can be integrated into reactivating beta-cells synthesis and macrophages synthesis (linear-beta forms), that can secrete IL-beta upon the stimulation of inflammatory molecules and by toxic irregular molecules, where, IL-beta can be reduced or inhibited through reactivating of the feedback of gamma subunits productions for restarting and reactivating TAZ basic pathway for rebuilding pyrimidine from purines and for rebuilding active signals transmission.

Macrophages are activated by the two glucocorticoids-subunits syntheses (gamma and Beta-subunit) through firstly the gammasubunits productions then followed by beta-subunits productions for promoting neutrophile activities through oxidative mRNAs productions for re-activating astrocytes functions.

Both macrophages and IL-beta responsible for building specified defense effects by recruiting other immune cells such as lymphocytes that act as anti-inflammatory tools and act as main tools for reactivating cells proliferations [10]. 
The increasing of the effectiveness of immunity can be firstly through the regulation of ATPase and COX-1 enzymes on inflammations for producing long fatty acids chains followed by synthetase effects for producing ROR-gamma (TAZ), then followed by synthase effects for producing fatty acyl-CoA-beta which will activate glucocorticoid subunits which will activate netrin-1, macrophages synthesis and neutrophiles synthesis, where neutrophiles are a multi fatty acylCoA-gamma +fatty-acyl-CoAsynthase (ROR-beta) active subunits +IL-beta for acting on inflammatory molecules that can contribute in the producing its own specified macrophages (linear forms),upon the effects of phospholipase for producing rounded circular forms of macrophages.

Both TAZ /YAP serve as a basic keys with regulatory roles in mesenchymal stem cells developments at several steps of osteochondrogenesis, where they promote progenitor cell expansion for third stage which depends on phospholipase function effects on both active glucocorticoid (gamma and Beta- glucocorticoid) for promoting the T-cells bio-synthesis (where T-cells basically depending on phospholipase effects (alpha-oxidations) on ROR-gamma and on ROR-beta (YAP Pathway) which is fatty acyl-CoA-synthase (beta subunits) for activating the proliferations processes through the synthesis of Alpha-subunits, and is acting as rheostats for terminal osteoblastic differentiation, (but not activate) where osteoporosis need the activation by phospholipase for activating the alpha-oxidations for proliferation and osteoblastic development.

At early stages of the development of YAP/ TAZ functions are differ at late stages of osteoblastic differentiation [11].

Where early processes of TAZ/YAP Pathway are differs from late processes due to the continually modifications of gamma and beta subunits by gamma and beta-oxidations that feed alpha-oxidations for proliferation through phospholipase effects for promoting osteoblastic development. Where, firstly gamma and beta-oxidation contributing in glucocorticoid subunits bio-synthesis, activating astrocytes, netrin-1 biosynthesis and neutrophile, but the stages of proliferations need to be activated by phospholipase effects for running alpha-oxidations for proliferation and T-cells bio-synthesis.

Hippo TAZ pathway is basic process for fatty acyl-CoA-synthetase synthesis upon the effects of synthetase enzymes on carbohydrate, on lipoproteins, on inflammations molecules for producing the active gamma-subunits through pyrimidine nucleotides synthesis for (maintenance) maintaining the basic balance and molecular numbers of purines and pyrimidines in interstitium fluid, and for cooperating with TCA cycles through adopting the purines synthesis (Synthesis from TCA cycles) by the pyrimidine synthesis for stimulating the acylCoA synthase production upon the effects of synthase on the active ROR-gamma subunits which later for IL-beta productions and for producing specified glial mRNA activities.

Stimulating the YAP signaling pathway is depending on firstly TAZ pathways through the production of the gamma subunits which will be the basis of the beta-subunits upon the effects of synthase, which are necessary for netrin-1 production for neutrophile repairs and activations which carry imp rules in anti- inflammatory effective processes.

The YAP (fatty acyl-CoA-synthase activities) Pathway is reflecting a critical role of the effect of Glial cell line-derived neurotrophic factor (GDNF) against $\mathrm{A} \beta$-induced inflammatory response in microglia [12]. Glucocorticoid's subunits (glucocorticoid-gamma \& beta) are acting as activating factor for the GDNF and for the membrane receptors DCC activations, where both glucocorticoids' subunits are considered as the basic receptors for activating netrin- 1 synthesis and for neuronal signals migration [13]. Where, glucocorticoids exert specific actions on expression of adhesion molecules on activated neutrophils [14].
The Hippo pathway and the RORs isoforms are fully identical and connected together in their origin and in their roles of activities and having the same identical biosynthesis pathways which started by the effects of synthetase enzyme effects for active gamma-subunits productions (TAZ signaling process) by gamma-oxidations process, followed by synthase effect (beta-oxidation) on fatty acyl-CoAgamma for producing fatty-acyl-CoA-beta subunits (YAP Pathway) for IL-beta, TNF-beta, glucocorticoid-beta, and thrompoxan-beta productions.

The oxidative-ligations processes for long active fatty acyl-CoA-beta production (beta-oxidations) will activate and regulate beta-cells repairs and cytokinesis-beta biosynthesis for linear forms of macrophages biosynthesis, and for active beta subunits as glucocorticoid-beta which regulate membrane receptors (which necessary for netrin-1 receptors synthesis) for neutrophile biosynthesis and repairs.

Both TAZ / YAP are depending on synthetase and on synthase activities functions respectively, and depending on ATPase +mitochondrial COX enzymes for producing long fatty acids chains, but, the productions of the primary four kind of protein kinases \{PSTCk (mTORC1 or PCK kinase), PSTTK, PSTGk, and PSTAK kinases $\}$ by FOX functions are basically depending on synthetase, synthase and phospholipase enzymes for producing the three RORs isoforms RORgamma (Gamma-oxidations) +ROR- beta(beta-oxidation) +RORalpha(alpha-oxidations) respectively.

Where due to the pro-nutrients-mTOR availabilities will be bind to FOX genes and will be affected firstly by ATPase and COX-1 enzymes for generating long fatty acids chains which will be followed by mitochondrial enzymes effects for generating the three RORs isoforms (ROR-gamma, beta, and alpha) synthesis upon the effects of the three mitochondrial anabolic enzymes the synthetase, synthase, and phospholipase enzymes respectively.

The Hippo signaling pathways considered as Gamma-oxidations (TAZ signaling pathway) followed by beta-oxidation (YAP signaling pathway) for ROR-gamma and ROR-beta isoforms productions synthesis and is highly depending on FOX genes activities which responsible for releasing different four kind of protein kinases which are basic for gamma oxidations then beta oxidations for releasing the active (Cytokine-beta), glucocorticoid-beta, netrin-1 then finally beta subunits can follow the alpha oxidations upon the effects of phospholipase for ROR-alpha productions for cells proliferation, for macrophages and for T-cells proliferations.

Where it has been considered that Hippo pathway is highly conserved kinase cascade that regulates cell proliferation, survival, mobility, stemness, and differentiation [15].

The blockage or inhibition or decreasing in Gamma-oxidations followed by decreasing in beta-oxidation will lead to accumulation of long fatty acids chains in cells tissue and can lead to severe hypoxia.

\section{Definition of Hypoxia}

Hypoxia is a condition in which the body or a region of the body is deprived of adequate oxygen supply which supposed to be through oxidative processes (RORs isoforms oxidative processes) which can affect all body. Hypoxias occur with any interruption to normal oxidative processes belongs to respiration [16], that reflect decreasing in oxidative mRNAs gamma and beta productions from astrocytes and inhibition in their improvements by glial cells.

Hypoxia started by full decreasing in COX or in ATPase enzymes Effects on the absorbed fatty nutrients- mTOR molecules and on inflammations molecules, that those molecules will not be analyzed properly then will reflect inhibitions in the production of long fatty acids chains that will lead to precipitation in and blockage in 
capillaries and may can reflect inhibitions in Gamma-oxidations, in beta-oxidation, then in alpha-oxidations.

Despite oxygen supply being given to patients with hypoxia, that will not give any progression due to their respiratory oxidative processes were full inhibited and blocked.

The three types of main basis of oxidations are: 1_Gamma-oxidations (result for releasing fatty acylCoA- synthetase upon the effects of synthetase enzymes on long fatty acids chains) (ROR-gamma isoforms), 2 2 then followed by beta-oxidation (fatty acyl-CoAsynthase upon the effects of synthase enzyme on gamma subunits) (ROR-beta isoforms), 3 then followed by alpha-oxidations (fatty acyl-CoA- phospholipase upon the effects of phospholipase on Betasubunit) (ROR-alpha isoforms).

The sudden inhibition in COX and ATPase activities followed by inhibition in respiratory oxidative processes (which mainly depends on Gamma-oxidations) that can be due to high temp effects or due to increasing in +ve molecules or due to full inhibitions in oxidative mRNA-gamma production lead to inhibition in glial cells activities.

The increasing in phosphorylated - ve bonding energy can increase mitochondrial effects for producing the oxidative mRNA-gamma by Gamma-oxidations on carbohydrate for hydrophobic acids synthesis for building active fatty-acyl-CoA synthetase subunits which will follow the synthase effects for building linear forms of fatty acyl-CoAsynthase subunits (oxidative mRNA-beta) for improvement by glial cells and increase respiratory cycles.

Due to internal (internal inflammation signals in vivo) or external agitation, the ATPase and mitochondrial COX enzymes will be secreted for acting on inflammation for producing the long fatty acids chains "where, COX de enzyme considered to be the first enzyme secreted by mitochondrial OPA1 inner membrane gene followed by synthetase, synthase then phospholipase respectively" (upon the signals created due to free protons and electrons from inflammatory molecules effects). That, long fatty acids chains which produced from effects of ATPase and cox enzymes will be followed by the effects of the synthetase enzyme for creating active signals through adopting purines by pyrimidine synthesis that will be the result of hydrophobic acids synthesis eg: Tyr, Leu, Cys, Gly.. etc for building ROR-gamma isoforms (gamma-subunits) synthesis including TNF-gamma, Thromboxanegamma, and glucocorticoid-gamma productions, which will follow the effects of synthase enzyme for beta-oxidations processes for ROR-beta isoforms synthesis, for TNF-beta synthesis, for IL-beta production," and for glucocorticoid-beta productions (for increasing oxidative mRNA-beta production through transcriptions), where both previous processes Gamma-oxidations and beta-oxidations can be considered as the basis for mRNA-gamma and mRNA-beta productions for astrocytes and brain glial activities modulations for completing and running oxidative processes, where oxidative mRNAs can be modified and improved by glial astrocytes cells which produce higher number of improved modified active mRNAs which have their own effective processes on inflammation molecules (as a type of anti-inflammatory effects), on adopting vision during light reduction, on increasing anti-inflammatory effectiveness, and on running respiratory cycles activities processes.

Where, the fatty acyl-CoA-synthetase and fatty-acyl-CoA-synthase (oxidative mRNAs) can be directed to be used as glucocorticoidgamma subunits and glucocorticoid-beta subunits for brain functions activities where will be used for reactivating (promote) astrocytes again (as feedback) and neutrophile repairs and modulations too. Neutrophil is the modulating essential tool for oxidative mRNAs productions for anti-inflammation and for astrocytes and brain reactivations.
Astrocytes have a robust enzymatic capacity for contributing in the glycolysis through cooperating to glycogenesis (TCA) cycles for adopting their results of TCA-purines productions by pyrimidines synthesis (as described previously upon the effects of synthetase enzyme $\&$ active gamma-subunits functions) where can be promoted by the biosynthesis of modified gamma-oxidative glial mRNAs (glial improved mRNAs), where, astrocytes have their own activities on lipid and on carbohydrate metabolism through its roles in re-activating Gamma-oxidations (upon the effects of synthetase) then followed by beta- oxidation effects for active beta-subunits productions and (betaoxidative glial mRNAs) for providing necessary support to the brain parenchyma for neuronal consumptions [17].

Where, Gamma-oxidations and beta-oxidation activities are providing the main supports (by oxidative mRNAs synthesis) to brain cells through active gamma-subunits productions (eg for modulating visions during light reduction), followed by beta-subunits productions (which necessary for IL-beta synthesis), and then followed by alphaoxidations for active alpha subunits productions (which is necessary for proliferations processes including T-cells biosynthesis which depending on phospholipase activities on previous subunits gamma and beta) upon the effects of phospholipase through converting linear forms to circular cells forms (through specific binding of amino acids which their types can varies according to oxidative mRNAs functions and tissues specificity) contains their necessary inner contents.

The neutrophile carry the basic role for astrocytes activities and repair where, the Polymorphonuclear neutrophils (PMN) are the most abundant class of white blood cells necessary for modulating astrocytes, and typically, the first type of leukocyte [18]

Reactivating Neutrophile (which is the basis for interstitium fluid efficiency) can be through reactivating necessary oxidative processes for producing oxidative mRNAs gamma and beta (by reactivating gamma oxidations and beta oxidations respectively for re-activating astrocytes, for brain glial activities, for macrophages resynthesis, then for T-cells synthesis (which upon availabilities of phospholipase enzymes which is necessary for proliferation processes).

The synthesis and modification of oxidative mRNAs start by the neutrophile modulations activities for astrocytes repair and for glial mRNAs (gamma and beta) improvements, where, it has been speculated that astrocytes are a source of ELR chemokines in the human central nervous system (CNS), in neurons and in astrocytes that can respond to those Chemokine [19].

The secreted protein Lipocalin-2 "LCN2" (modulated oxidative mRNAs) is an autocrine modulator of the functional polarization by astrocytes due to immune or inflammatory stimuli and that Lipocalin-2 (LCN2) could be targeted therapeutically to dampen pro-inflammatory astrocytic activation and related pathologies in the central nervous system (CNS) [20]

Synthetase is the basic effective tool for modulating prostaglandins, for anti-inflammations, and for improving phospholipid membranes in availability of phospholipase. Phospholipid (phospho-lipo-protein) molecules are designed as protective biological molecules for cells membrane protections from temperature variations effects, from viral effects, and from normal catabolic processes effects (except the ATPase and COX effects that have the ability to analyze phospholipid for producing prostaglandins).

The number of detergent molecules bound to purified membrane proteins is dependent on the physicochemical properties of the detergents and on the hydrophobic lipid-accessible protein surfaces [21]. i.e., synthetase activities for hydrophobic acids synthesis is necessary for phospholipid synthesis and binding to cells membranes for filtration and protection. 
Due to inflammatory stimulated factors, the phospholipids membranes will be degraded by the effects of ATPase and COX enzymes on phospholipid and on inflammations molecules to produce fatty acids chains which upon synthetase effects will produce gamma subunits (long fatty-acyl-CoA-gamma isoforms), which upon the following effect of synthase will produce beta subunits (upon beta- oxidations) which can be the modulator for glucocorticoid-beta synthesis and neutrophile modulations.

The effects of synthetase on long fatty acids responsible for producing ROR-gamma (fatty-acyl-CoA- synthetase) and active signals which followed by the effect of synthase enzyme as the second step for producing ROR-beta isoforms (beta-subunits) that can be the main steps for linear macrophages synthesis (beta-macrophages), for the IL-beta synthesis and for neutrophile biosynthesis through netrin- 1 synthesis. Neutrophiles include IL-beta where, neutrophile modulate the oxidative mRNAs (upon gamma and beta oxidations) productions.

Due to gamma oxidations that increase carbohydrate metabolic processes, the adoptions of purines and pyrimidine synthesis will be increased (upon the effects of synthetase enzymes with cooperation with Tri-carboxylic acid "TCA" cycles) with the increasing of the releasing of free protons and electrons which create signals for reactivating migrations and stimulate other cells for modulating neuron metabolic processes and brain by oxidative- mRNAs productions for astrocytes and brain activities that can re-activate signals again through feedback for RORs isoforms productions.

The OPA1 inner membrane gene contains specific sequences in orders of arrangement of (mito-enz) mitochondrial enzymes that can express firstly COX enzymes for degradation the nutrient-mTOR and inflammations molecules followed by the three of mitochondrial enzymes which are responsible for expressing RORs isoforms and oxidative mRNAs subunits, that firstly started by effect of synthetase enzymes followed by synthase efforts and ended up by phospholipase Effects, that each of those mitochondrial enzyme in OPA1 gene can be affect and release in orders its own isoform and subunits, and all are depending on each other's in order respectively.

Induction of prostaglandin "PGE2" biosynthesis by IL-1 $\beta$ is depending and mediated by protein-kinases $\mathrm{C}$ (PKC which originally produced by FOX gene activity) and mitogen-activated protein kinases Is fully connected and related to previous step of gamma-oxidations processes then followed by synthase effects for (beta-oxidations) processes for producing IL-beta for anti-inflammation processes upon inflammation stimuli and upon releasing pro-inflammation molecules (which firstly upon the effects of synthetase).

Fatty acyl-CoA-Synthetase subunits (gamma-subunits) and phorbol ester are having the same origin and same activities. Upon the stimulations of Astrocytes by phorbol ester (PMA) will be followed by IL-1 $\beta$ synthesis and will reflect increasing in carbohydrate digestion and metabolism and will reflect increasing in purines and pyrimidine level adoption for hydrophobic acids synthesis.

The PKC membrane are the main compositions of astrocytes, where the increasing in PMA can analyse and break PKC membrane for producing gamma subunits and for pyrimidine synthesis, where PMA can be considered as the basis for IL-beta productions and has the function of creating and increase signals migrations due to its activities, where PMA is playing the same activities and functions roles as the acyl- CoA synthetase activities and activate gamma-oxidations for gamma subunits productions followed by beta-oxidation for betasubunits productions which can repairs beta cells., and can activate IL-beta, TNF-beta, and for Thromboxane-beta productions.

The highly effects of PMA gamma oxidations on PKC membrane will analyze its contents result of releasing pro-inflammatory molecules and converting some purines to pyrimidines nucleotides (reflect increasing in glucose metabolism) which create active signals through creating its gamma-subunits.

The reduction or the downstream of PMA will reduce the prostaglandins PGE2 productions and will reduce the IL-beta productions too upon reductions in Gamma-oxidations. Where, the PKC-depleted astrocyte cultures by PMA no longer responded to PMA or IL-1 (indicating that PKC has active sites responds to and activated only by PMA), and the The ablation of the effects of PMA and IL-1 $\beta$ on PGE2 production, likely results from down-regulation of phorbol ester sensitive-PKC isoenzymes [22].

Astrocytes are created structures activated by neutrophils through gamma oxidations followed by beta oxidations processes for extending the oxidative activities by producing mRNAs (gamma and beta) for brain activities that can be extended to cells proliferations upon phospholipase activities for T-cells proliferations.

The astrocytes synthesis and repair firstly activated by gamma oxidations followed by beta oxidations for producing mRNAs gamma upon phorbol ester (PMA) activities.

Astrocytes express various G-protein Coupled Receptors (GPCRs) and depending on ser/Thr kinases avaliablities and on synthetase enzyme for producing acyl-CoA gamma subunits followed by acylCoA beta productions, that can be activated by neurotransmitters, by neuropeptides (neuro-hydrophobic amino acids). Where, astrocytes express all three types of $\beta$-adrenergic receptors $(\beta 1, \beta 2$, and $\beta 3)$ [23].

The Fatty acid and phorbol ester-mediated interference of mitogenic signaling via novel protein kinase $\mathrm{C}$ isoforms in pancreatic beta-cells for firstly producing the acyl-CoAs-synthetase(gamma-subunits) which followed by acyl-CoA-synthase production, where the phorbol esters necessary for producing PKC upon its effect on long fatty acids for producing pyrimidines for gamma subunits synthesis and then for IL- 1beta synthesis upon synthase effect [24].

Where, phorbol ester (PMA) represents the activities of acyl-CoA synthetase subunits (gamma-subunits) which has strong effects on carbohydrate metabolism and induce active signals which stimulate the gamma-subunits productions, and then beta subunits productions and considered as is carrying the basic roles for pro-inflammation synthesis and for increasing the anti-inflammatory processes in the availabilities of IL-beta synthesis, where PMA is responsible for ILbeta synthesis for astrocytes repairs, and for the two glucocorticoids subunits synthesis.

Note, Phorbol ester (PMA) is employed in biomedical studies to create signal transmission activities due to its activities for creating free protons and free active electrons for re-activating signals for stimulating biological cycles eg: its effects on carbohydrate, on sugar, and on fatty acids chains for pyrimidine synthesis that can cooperate with TCA cycles for adopting sugar purines through pyrimidine synthesis. Where, PMA has strong effects on carbohydrate digestion for pyrimidine synthesis from purines for hydrophobic fatty and amino acids synthesis [25].

Also, PMA enhanced the synthesis of proteoglycans (PG)which is the basic for collagen synthesis and depending on Ser and Gly synthesis and availabilities (where, in case of deficiency in PMA activities and in Ser deficiency will lead to joints pain and cartilage erosion due to lack of PG synthesis) where the stimulation of the PMA synthesis reflects an increase in proteoglycans (PG) protein moiety productions and tend to an increase in the glycation process [26]

Where, the increasing in the effects of PMA on fatty acids chains will increase fatty glycoprotein synthesis and will increase the spontaneous non-enzymatic reactions of free reducing sugars with free amino groups giving glycan molecules. 
Where the increasing in phorbol ester can reflect increasing in proteoglycan synthesis through the effects of synthetase on carbohydrate and on purines for pyrimidine synthesis followed by its effect on fatty acids through enhancing the production of pyrimidine nucleotides for hydrophobic acids synthesis for producing acyl-CoAsynthetase gamma subunits productions which can act spontaneously for free amino acids synthesis.

The effects of phorbol esters can be considered as has the roles in activating irreversible Diacylglycerols (DAG) through its effects on long fatty acids chains for producing irreversible DAG molecules due to pyrimidine synthesis which can participate in forming active irreversible PKC membrane [27].

Where, due to the conversion of purines to pyrimidine nucleotides will cause irreversible process for hydrophobic acids synthesis upon the effects of PMA on protein-glycan and on carbohydrate. Also, the active phorbol esters can act as enzyme to generate irreversible pyrimidine synthesis for rebuilding PKC-membrane complex.

Also, the activation of protein kinase $\mathrm{C}$ can activate phorbol esters sufficiently to promote NOS induction [28]. Gamma-oxidations can activate phorbol esters which has the responsibility for increasing cellular signals activities which promote cellular migration for extending to the interleukin beta synthesis, where Phorbol Ester Modulates Interleukin 6- and Interleukin 1-regulated Expression [29].

The PMA and acyl-CoA synthetase (acyl-CoA-gamma) are having the same origin and are so necessary for inducing pyrimidines nucleotides for hydrophobic amino acids synthesis for IL-beta synthesis and for astrocytes repair through the synthesis of PKC membrane, and also indicates the value and necessity of acyl-CoA-gamma subunits as the basis of several necessary metabolic processes including the acylCoA- synthase (beta-subunits) productions for neutrophile repair, for activating astrocytes, and for netrin-1 synthesis, where, netrin-1 play imp roles in development of lungs and pancreas beta-cells, and described as neuronal guidance and have bifunctionally roles 1st is catabolic processes which is basis for pyrimidine synthesis and for IL-beta production which is anabolic effect for increasing antiinflammatory tools and processes. The netrin-1 activate growth cone collapse, and can induces both of protein degradation and rapid growth cone collapse activation, where Sema3A-induced growth cone. collapse, requires protein synthesis and p42/p44 (which depend on synthetase activities) [30].

Astrocytes can be stimulated by reduction in light that can activate gamma-oxidations that can be the results in creating signals and G-protein development for modifying optical development and modify width of eye pupil and according to light effect, where, Optogenetic activation of Gq signaling in astrocytes yields stimulations [31].

The stimulation of light reduction to astrocytes indicate the stimulations firstly of the gamma-oxidations by astrocytes for activating their phorbol ester subunits or acyl-CoA synthetase subunits for producing their imp gamma subunits (p38, p42) which are necessary for starting the increasing of the IL-beta and other beta subunits upon the effects of beta oxidations by synthase enzyme and can create more of effective signals which can modify vision and produce the two types of mRNAs gamma and beta for activating glial and increase the modification of mRNAs for brain activities and can be the results of IL-beta and netrin-1 improvements and G-protein improvements.

Plasmids is double stranded subunits gamma-subunits and Betasubunit that each is running own oxidative processes, where basically plasmid are having the basic origin of the biosynthesis of gamma and beta subunits (fatty-acyl-CoA gamma and beta subunits) in vertebrates (fatty-acyl-CoA synthetase and synthase), that originally depend on synthetase then synthase enzymes in their origin, that can found as linear structures or in circular forms depends on their composition from amino acids. Types of plasmids depends on their content from hydrophobic amino \& fatty acids.

In their double subunits chain that exist in bacteria as extrachromosomal subunits (gamma \& beta) that have self-replicating characters. Note, bacterial plasmids subunits are able in vivo to run the glucocorticoid cycles activities for activating netrin-1 and for DCC synthesis that bacteria produce its own subunits for running their own pathological pathways. That I can consider that excretions from glucocorticoids subunits can be improved in vitro environments to be plasmids that their classes can be due to +ve bonding content and due to their amino acids content in specific arrangements.

Gamma oxidations inhibit TLR4, but beta-oxidations followed by alpha-oxidations will activate TLR4 and T-cells synthesis and functions. Toll-like receptor 4 (TLR4), is activated by lipopolysaccharide (LPS) where, LPS is activating Gamma- oxidations process results of producing fatty-acyl-CoA synthetase (gamma-subunits) which under synthetase effects will produce fatty-acyl-CoA synthase (betasubunits) which necessary for producing IL-beta that is promote TLR4 biosynthesis (for T-cells biosynthesis) to initiate anti-inflammatory responses and oxidative stress (which includes three types of oxidations for producing the three types of active subunits gamma, beta, and alpha). Oxidative processes firstly start by ATPase and COX enzyme lead to some irregular intermediates subunits productions, in case of deficiency in mitochondrial enzymes synthetase and synthase that will reflect deficiency in pyrimidines synthesis and deficiency in adopting blood glucose or purines that will lead to irregular or mutated subunits productions that will not be able to produce IL-beta and TLR4transmembrane which is necessary for T-cells activities that can clear invading pathogens.

The lipopolysaccharides (LPSs) are reported to be TLR4 antagonists, and at the same time considered as preventing lung cancer that LPS-Rs is activating the 1st step of Gamma-oxidations for producing the basic gamma-subunits for generating Beta-subunit for generating Alphasubunits for TLR4 transmembrane synthesis and for activating their own RAW264.7 cells.

That LPSs molecules activate Gamma-oxidations for fatty-acylCoAsynthetas protection (ROR gamma) followed by beta-oxidation then followed by alpha-oxidations will activate TLR4 synthesis that activate T-cells function for their tissues.

Where, Activation of RAW264.7 cells with a lipopolysaccharide (LPS) specific for the TLR4 receptor will involve activating the three RORs isoforms activities (specifically ROR-alpha activities that generate RAW264.7 cells with its active TLR4 transmembrane) and activating beta and alpha-oxidations processes necessary for cells proliferations, causes a large increase in cellular sphingolipids [32].

LPS is the basic that firstly promote gamma oxidations through synthetase functions which will follow synthase effects for Beta-subunit productions which will promote TLR4 synthesis upon phospholipase effects (alpha-oxidations) on Beta-subunit for initiating those types of transmembrane which can increase T-cells activities into brain. TLR4 gene is a beta subunit (originated from gamma-subunits) that promoted by fatty-acyl-CoA-synthase (Beta-subunits "beta-oxidations "), that its composition included both fatty gamma and beta subunits that able to activate both gamma and beta oxidations.

Where, the activation of TLR4 transmembrane through activating beta-subunits (beta-oxidations) followed by alpha-oxidations upon the effects of phospholipase leads to activating intracellular signaling pathway NF- $\mathrm{KB}$ and anti-inflammatory cytokine productions. But activation through fatty-acyl-CoA synthetase (gamma oxidations) will lead to attenuate the production of TLR4 till will complete its Gammaoxidations for producing gamma-subunits then will follow betaoxidations and alpha-oxidations for TLR4 synthesis upon the effects 
of phospholipase functions for activating RAW264.7 cells and T-cells.

That due to some of disease conditions, the TLR4 transmembrane can be analyzed due to high expression of gamma oxidations lead to free up own main amino acids contents by effect of synthetase enzyme, as eg of LPS can activate Gamma-oxidations for acting on lipid, on carbohydrate, on lipoproteins, and on trans-membranes for producing gamma-subunits which will follow the rest of mitochondrial enzymes for producing beta and alpha subunits for TLR4 synthesis which activate T-cells functions. That, it has been reported that LPS-Rs, through TLR4 antagonism, significantly inhibit TLR4 mediated inflammatory [33].

BCL6 which regulate lipid metabolism is promoted by gammaoxidations, which is considered as fatty- acyl-CoA-synthetase (gamma-subunits) which upon beta-oxidation will activate Betasubunit which is necessary for macrophages activities.

BCL6 biosynthesis is promoted by proper Gamma-oxidations by synthetase enzyme on fatty acids chains for producing BCL6, where, BCL6 is considered as fatty-acyl-CoA-synthetase (gamma-subunits) that necessary and involved in fat and in carbohydrate metabolism, which upon beta-oxidation will activate Beta-subunit which is necessary for activating beta-cells, for autophagy synthesis, and is believed that participate in the regulation of sugar with TCA cycles, and promote uncoupled respiration through increasing active sites in gamma-subunits for carrying oxygen.

BCL6 drives a gene expression program that promotes survival, fatty acid oxidation (through activating fatty-acyl-CoA-synthetases active subunits which adopt environmental warmth), and uncoupled respiration. BCL6 is specifically required for maintaining thermogenic fitness during adaptation to environmental warmth [34].

Where, degradation or mutations in BCL6 or BCL10 will promote B-cell lymphoma/leukemia 10 can stimulate K63 poly-ubiquitination and inhibit fatty acid oxidation led to accumulation of fatty acids on inner blood vessels walls and inhibit uncoupled respiration activities.

The Dysfunction in synthetase sequences in OPA1 inner mitochondrial membrane which is responsible for lipid and carbohydrate metabolism for converting purines to pyrimidine and building migrating active signals that activate the adopting vision will lead to dysfunction in BCL6 genes, synthesis, and lead to optic neuropathy.

That the dysfunction of mitochondrial OPA1 gene in ADOA is thought to be associated with apoptosis of retinal ganglion cells leading to optic neuropathy [35].

That activity which responsible for cells proliferation and for converting linear-RNAs forms to circular active RNA is controlled by Ser\&Arg linkages and Thr (ACC) \& Trp (TGG) linkages \& Thr (ACA) \& Cys (TGT) linkages, where phospholipase is necessary to connect and bind those amino acids and convert linear-RNAs to active circular-RNAs forms.

BCL6 is necessary for activation by inducing interactions between conventional dendritic cells (DCs) and T cells, Where B cells support the expansion of Bcl6, that B-cell produce beta-subunits which supports Bcl6 (gamma-subunits) through its functions for running gamma-oxidation for activating T-cells by building the interactive between dendritic and T cells.

(ROR- $\gamma$ ) synthesis is depending on synthetase enzymes effects on fatty acids chains and is the basic activator key for transcription factors necessary for Bcl6 productions and for re-activating Th17 cell.

\section{Conclusion}

OPA1 is basis for macrophages biosynthesis, for glucocorticoids subunits productions, for neutrophile proper repair, and for reactivating RAW264.7 cells and T-cells and regulations, where OPA1 contain COX enzyme sequence followed by synthetase, then synthase, then phospholipase sequences respectively in its biological active chain.

fatty-acyl-CoA-synthetase (ROR-gamma) is the biological structures and composition for gamma- Subunits, for glucocorticoid-gamma subunits, for Phorbol ester (PMA), and for oxidative mRNA-gamma which necessary for glial cells activities, and for Bcl6 is carrying the same biological compositions of fatty-acyl-CoA-synthetase where its biosynthesis depends on synthetase effects on fatty long chains and on FOX gene functions. While fatty-acyl-CoA-synthase is the main composition of Beta-subunit, for glucocorticoid beta, for TLR4 transmembrane synthesis and for oxidative mRNA-beta production necessary for astrocytes reactivities.

Hippo pathway and The RORs isoforms biosynthesis are comprising the Ser /Thr kinase cascade that are fully depending on proper FOX gene function and pathways, where, both of Taz pathway and RORgamma (fatty-acyl-CoA synthetase) subunits synthesis are having the same origin of pathways and activities for rebuilding pyrimidine from purines upon their effect on carbohydrate, on fatty acids, and on sugar molecules for hydrophobic acids synthesis for building active gammasubunits which used for Beta-subunit synthesis upon synthase effects.

Glucocorticoid-gamma has anti-inflammatory activities on inflammations for producing pro- inflammatory subunits due to the effects of acyl-CoA synthetase (gamma subunits) on inflammations for analyzing their contents for starting anti-inflammatory pathways activity, while glucocorticoid-beta is attributed to the repression of pro-inflammatory subunits through signal transduction by their steroid beta-subunits (beta oxidations). That glucocorticoid can have two basics subunits.

\section{a) Glucocorticoid-gamma,}

b) Glucocorticoids-beta, that both of glucocorticoid subunits are having roles of increasing anti- inflammatory pathways.

Hypoxia occur through interruption and decreasing in normal oxidative processes belongs to respiratory pathways, that reflect full decreasing in oxidative-mRNAs (gamma and beta mRNAs productions) and inhibition in their improvements by glial cells and reflect a full decreasing in astrocytes activities and in glucocorticoids subunits productions and functions.

Neutrophil is the modulating essential tool for oxidative mRNAs productions which are necessary for anti-inflammation and for astrocytes and brain re-activations and necessary for recoveries from hypoxia. The gamma-oxidation is basis for producing fattyacyl-CoA-synthetase which is necessary for running beta-oxidations for producing (Beta-subunit) linear TLR4 which followed by alphaoxidations for full activating TLR4 and T-cells synthesis through phospholipase effects and regulations.

BCL6 synthesis is regulating lipid, and involved in carbohydrate metabolism, and is promoted by gamma-oxidations, where BCL6 is considered as fatty-acyl-CoA-synthetase (gamma-subunits) which upon beta-oxidation will activate Beta-subunit and B-cell which is necessary for promoting anti- inflammatory pathways and autophagy activities.

Antioxidants are substances that are easy activate fatty-acyl-CoAsynthetase synthesis and then stimulate fatty-acyl-CoA-synthase synthesis and then transmembrane synthesis for protecting cells from free radicals, which play a role in heart disease, cancer and other diseases. Where Free radicals are molecules produced when your body breaks pro nutrients-mTOR by COX and ATPase spontaneously through agitation or when you're exposed to high heated atmosphere or due to high tobacco smoke or exposed to acute radiation. 
As eg of vit $\mathrm{C}$ that promote TCA cycles that will stimulate the mitochondrial synthetase to act on fatty acids, on carbohydrate, on sugar molecules, and on extra purines from TCA cycles for necessary pyrimidine synthesis for hydrophobic acids synthesis for building first gamma-subunits which will be the basis for neutrophile repairs, for the two glucocorticoids subunits synthesis from neutrophile and from ROR-gamma then from ROR-beta for producing glucocorticoidgamma then glucocorticoid-beta respectively, which in neutrophiles can be considered as oxidative RNA-gamma and oxidative RNA-beta respectively for running gamma-oxidation and beta-oxidation where are both considered as anabolic processes for reactivating astrocytes for brain activities and considered as basic processes for regulating the adoption of estrogen and Androgen's hormones in vivo where both are depending on those two imp previous oxidations and on fox Ser/Thr cascade functions where in case of increasing androgen will enhance Gamma-oxidations activity for reproducting pyrimidine for resynthesis Ser amino acids for running estrogen biosynthesis.

\section{Conflict of Interest}

The Author declare that the research work has been conducted in the absence of any commercial or financial relationships, that could be construed as a potential conflict of interest.

\section{References}

1. Ging J, Yu S, H Zhao, Sun X, Li X, et al. (2017) The transcriptional coactivator TAZ regulates reciprocal differentiation of T.sub.H17 cells and T.sub.reg cells. Nat Immunol 18(7): 800-812.

2. Ecouer F, Weiss J, Kaupmann K, Hintermann S, Orain D, et al. (2019) Antagonizing Retinoic Acid-Related-Orphan Receptor-Gamma Activity Blocks the T Helper 17/Interleukin-17 Pathway Leading to Attenuated Pro-inflammatory Human Keratinocyte and Skin Responses. Front Immunol 10: 577

3. Rausch V, Hansen G C (2020) The Hippo Pathway, YAP/TAZ, and the Plasma Membrane. Trends Cell Biol 30(1): 32-48.

4. Lourdes Rocamora Reverte, Holger M Reichardt, GJan Wiegers, Andreas Villunger (2017) T-cell autonomous death induced by regeneration of inert glucocorticoid metabolites. Cell Death \& Disease 8: e2948.

5. Cindy Strehl, Lisa Ehlers, Timo Gaber, Frank Buttgereit (2019) Glucocorticoids-All-Rounders Tackling the Versatile Players of the Immune System. Front Immunol 10: 1744.

6. Quatrini L, Ugolini S (2021) New insights into the cell- and tissuespecificity of glucocorticoid actions. Cell Mol Immunol 18(2): 269278.

7. Simiao Xu, Yangyang Liu, Ruixiang Hu, Min Wang, Oliver Stöhr, et al (2020) TAZ inhibits GR and coordinates hepatic glucose homeostasis in normal physiologic states.

8. Mo JS, Meng Z, Kim YC, Park HW, Hansen CG, et al. (2015) Cellular energy stress induces AMPK-mediated regulation of YAP and the hippo pathway. Nat Cell Biol 17(4): 500-10.

9. Wang W, Xiao ZD, Li X, Aziz KE, Gan B, et al. (2015) AMPK modulates Hippo pathway activity to regulate energy homeostasis. Nat Cell Biol 17: 490-499.

10. Vijaykumar S Meli, Hamza Atcha, Praveen Krishna Veerasubramanian, Raji R Nagalla, Thuy U Luu (2020) YAP-mediated mechanotransduction tunes the macrophage inflammatory response. Science Advances 6(49): eabb8471.

11. Heinrich Kovar, Lisa Bierbaumer, Branka Radic-Sarikas (2020) The YAP/TAZ Pathway in Osteogenesis and Bone Sarcoma Pathogenesis. Cells 9(4): 972.

12. Jie Qing, Xiaoheng Liu, Quan Wu, Mengjie Zhou, Yuwei Zhang (2020) Hippo/YAP Pathway Plays a Critical Role in Effect of Glial cell-derived neurotrophic factor (GDNF) Against $A \beta$-Induced Inflammation in Microglial Cells. DNA Cell Biol 39(6): 1064-1071.
13. Fabien Llambi, Frédéric Causeret, E Bloch Gallego, P Mehlen (2001) Netrin- 1 acts as a survival factor via its receptors UNC5H and DCC. EMBO 20(11): 2715-2722.

14. János G Filep, Aline Delalandre, Yves Payette, Éva Földes Filep (1997) Glucocorticoid Receptor Regulates Expression of L-Selectin and CD11/CD18 on Human Neutrophils. Circulation 96(1): 295-301.

15. Xie Z, Wang Y, Yang G, Jing H, Zhu L, et al. (2021) The role of the Hippo pathway in the pathogenesis of inflammatory bowel disease. Cell Death Dis 12(1): 79.

16. (Medical Editor: Charles Patrick Davis, MD, PhD Reviewed on $3 / 29 / 2021$

17. Mitsuhiro Morita, Mami Noda, Marko Kreft, Robert Zorec, Nina Vardjan, et al. (2019) Metabolic Plasticity of Astrocytes and Aging of the Brain. Int J Mol Sci 20(4): 941.

18. Xie L, Poteet EC, Li W, Scott AE, Liu R, et al. (2010) Modulation of polymorphonuclear neutrophil functions by astrocytes. J Neuroinflammation 7: 53

19. W Lu, A Maheshwari, I Misiuta, SE Fox, N Chen (2005) Neutrophilspecific chemokines are produced by astrocytic cells but not neuronal cells. Brain Res Dev Brain res 155(2): 127-134.

20. Eunha Jang, Jong Heon Kim, Shinrye Lee, Jae Hong Kim, Jung Wan Seo, et al. (2013) Phenotypic Polarization of Activated Astrocytes: The Critical Role of Lipocalin-2 in the Classical Inflammatory Activation Of Astrocytes. J Immunol 191(10): 5204-5219.

21. Eduardo Molina Holgado, Carmen Guaza, S Ortiz, F Molina Holgado (2000) Induction of COX-2 and PGE2 biosynthesis by IL-1 $\beta$ is mediated by $\mathrm{PKC}$ and mitogen-activated protein kinases in murine astrocytes. Br J Pharmacol 131(1): 152-159.

22. Hüseyin Ilgü, Jean Marc Jeckelmann, María Salomé Gachet, Zöhre Ucurum, Rajendra B, et al. (2014) Variation of the Detergent-Binding Capacity and Phospholipid Content of Membrane Proteins When Purified in Different Detergents. Biophys J 106(8): 1660-1670.

23. Zhiwen Zhou, Yuji Ikegaya, Ryuta Koyama (2019) The Astrocytic cAMP Pathway in Health and Disease. Int J Mol Sci 20(3): 779.

24. C E Wrede, L M Dickson, M K Lingohr, I Briaud, C J Rhodes (2003) Fatty acid and phorbol ester-mediated interference of mitogenic signaling via novel protein kinase $\mathrm{C}$ isoforms in pancreatic beta-cells (INS-1). J Mol Endocrinol 30(3): 271- 286.

25. P A MacLennan, A McArdle, RH Edwards (1991) Acute effects of phorbol esters on the protein-synthetic rate and carbohydrate metabolism of normal and mdx mouse muscles. Biochem J 275(2): 477-483.

26. Thiébot B, Langris M, Bonnamy PJ, Bocquet J (1999) Activation of protein kinase $\mathrm{C}$ pathway by phorbol ester results in a proteoglycan synthesis increase in peritubular cells from immature rat testis. Biochim Biophys Acta 1426(1): 151-167.

27. Bazzi MD, Nelsestuen GL (1989) Differences in the effects of phorbol esters and diacylglycerols on protein kinase C. Biochemistry 28(24): 9317-9323.

28. S Hortelano, A M Genaro, L Bosca (1993) Phorbol esters induce nitric oxide synthase and increase arginine influx in cultured peritoneal macrophages. FEBS lett 320(2): 135-139.

29. Heinz Baumann, Hadar Isseroff, Gerald P Jahreis, J J Latimer (1988) Phorbol Ester Modulates Interleukin 6- and Interleukin 1-regulated Expression of Acute Phase Plasma Proteins in Hepatoma Cells. J Biol Chem 263(33): 17390-17396.

30. Douglas S Campbell, Christine E Holt (2003) Apoptotic Pathway and MAPKs Differentially Regulate Chemotropic Responses of Retinal Growth Cones. Neuron 37(6): 939-952.

31. Connor D Courtney, Courtney Sobieski, Charu Ramakrishnan, Robbie J Ingram, Natalia M Wojnowski, et al. (2021) Optogenetic activation of $\mathrm{Gq}$ signaling in astrocytes yields stimulation-specific effects on basal hippocampal synaptic excitation and inhibition. 
32. Sims K, Haynes CA, Kelly S, Allegood JC, Wang E, et al. (2010) Kdo2-lipid A, a TLR4-specific agonist, induces de novo sphingolipid biosynthesis in RAW264.7 macrophages, which is essential for induction of autophagy. J Biol Chem 285(49): 38568-38579.

33. Jurga AM, Rojewska E, Makuch W, Mika J (2018) Lipopolysaccharide from Rhodobacter sphaeroides (TLR4 antagonist) attenuates hypersensitivity and modulates nociceptive factors. Pharm Biol 56(1): $275-286$.
34. Vassily I Kutyavina, Ajay Chawlaa (2019) BCL6 regulates brown adipocyte dormancy to maintain Thermogenic reserve and fitness. Physiology 116(34): 17071-17080.

35. Buono LM, Foroozan R, Sergott RC, Savino PJ (2002) Is normal tension glaucoma actually an unrecognized hereditary optic neuropathy? New evidence from genetic analysis. Curr Opin Ophthalmol 13: 362-370. 